\section{ESTRATÉGIA EDUCATIVA SOBRE MANEJO DE RESÍDUOS SÓLIDOS DE SAÚDE NA UNIDADE DE TERAPIA INTENSIVA}

\author{
Educational intervention on medical waste in the Intensive \\ Care Unit
}

Estrategia educativa para el manejo de residuos sólidos sanitarios de la Unidad de Cuidados Intensivos

\section{RESUMO}

Objetivo: Avaliar a ação de estratégia educativa sobre o conhecimento de profissionais que atuam em Unidade de Terapia Intensiva quanto à segregação de resíduos sólidos de saúde. Métodos: Estudo experimental desenvolvido em 2015, em um hospital público de Fortaleza, Ceará, Brasil, com 41 profissionais de saúde submetidos à estratégia educativa, com álbum seriado sobre resíduos sólidos de saúde, comparando-se seu conhecimento com a realização de pré e pós-teste em formulário adaptado da literatura. Para a análise estatística, utilizou-se teste $t$, binomial e $\mathrm{p}<0,05$. Resultados: Com a realização da estratégia educativa, observouse diferença estatisticamente significante na média de acertos entre o pré-teste $(19,6 \pm 3,2)$ e pós-teste $(24,1 \pm 2,8), p=0,001$. A categoria sobre os resíduos comuns foi a que teve um maior número de erros entre os profissionais de saúde. Conclusão: A estratégia educativa proporcionou o aumento do conhecimento dos profissionais sobre a segregação dos resíduos, o que poderá favorecer a redução de acidentes ocupacionais de saúde e a redução dos gastos com materiais.

Descritores: Resíduos de Serviços de Saúde; Capacitação em Serviço; Unidades de Terapia Intensiva.

\section{ABSTRACT}

Objective: To assess the effect of an educational intervention on the knowledge of Intensive Care Unit professionals regarding medical waste. Methods: Experimental study conducted in 2015 at a public hospital of Fortaleza, Ceará, Brazil, with 41 healthcare professionals who were submitted to an educational strategy that used a flipchart on solid waste, comparing their knowledge before and after a test using a formulary adapted from the literature. The $t$ test and binominal test were used for statistical analysis with $p<0.05$. Results: After the educational intervention, there was a statistically significant difference in the mean score between pre-test $(19.6 \pm 3.2)$ and post-test $(24.1 \pm 2.8), p=0$,001.The category on common waste was the one with the highest number of errors among healthcare professionals. Conclusion: The educational strategy enhanced healthcare professionals'knowledge on the medical waste, which may favor the reduction of occupational accidents in health care and material expenses.

Descriptors: Medical Waste; Inservice Training; Intensive Care Units.
Artigo Original

Bruna Bianchi Bilo ${ }^{(1)}$

Lívia Moreira Barros ${ }^{(1)}$

Leonardo Alexandrino da Silva ${ }^{(1)}$ Francisca de Melo Beserra ${ }^{(2)}$ Joselany Áfio Caetano(1)

1) Universidade Federal do Ceará - UFC Fortaleza (CE) - Brasil

2) Hospital Universitário Walter Cantídio HUWC - Fortaleza (CE) - Brasil

Recebido em: 25/02/2016

Revisado em: 30/03/2016 Aceito em: 17/06/2016 


\section{RESUMEN}

Objetivo: Valorar la acción de estrategia educativa sobre el conocimiento de los profesionales de la Unidad de Cuidados Intensivos respecto la segregación de residuos sólidos sanitarios. Métodos: Estudio experimental desarrollado en 2015 en un hospital público de Fortaleza, Ceará, Brasil, con 41 profesionales sanitarios que participaron de la estrategia educativa con álbum ilustrativo sobre residuos sólidos sanitarios comparándose su conocimiento a través de pre y pos test con un formulario adaptado de la literatura. Se utilizó la prueba $t$, binomial y $p<0,05$ para el análisis estadístico. Resultados: De la realización de la estrategia educativa se observó una diferencia estadísticamente significativa de la media de aciertos entre el pre $(19,6 \pm 3,2)$ y el pos test $(24,1 \pm 2,8), p=0,001$. Hubo más errores entre los profesionales sanitarios para la categoría de los residuos comunes. Conclusión: La estrategia educativa proporcionó más conocimientos a los profesionales sobre la segregación de los residuos lo que puede llevar a una reducción de accidentes ocupacionales de salud y de los gastos con materiales.

Descriptores: Residuos Sanitarios; Capacitación en Servicio; Unidades de Cuidados Intensivos.

\section{INTRODUÇÃO}

Ações de promoção da saúde visam atuar sobre os determinantes da saúde, tais como fatores sociais, econômicos, culturais e ambientais, com o objetivo de implementar mudanças nas dimensões que afetam a sociedade como um todo e prejudicam a qualidade de vida da população ${ }^{(1,2)}$. Nessa perspectiva, o crescimento tecnológico e industrial acelerado trouxe consequências importantes para a sociedade contemporânea e para o meio ambiente. $\mathrm{O}$ aumento da produção de resíduos é consequência direta da rápida urbanização e crescimento populacional, necessitando de tratamento e gestão especializada. A indiscriminada produção de resíduos causa grave desequilíbrio ambiental, e o descarte inadequado desses materiais coloca em risco os recursos naturais e a qualidade de vida da sociedade e das futuras gerações ${ }^{(3,4)}$.

Entre os vários tipos de resíduos existentes, destacamse os Resíduos de Serviço de Saúde (RSS), que incluem todo o lixo produzido pelos serviços de saúde, centros de pesquisa e laboratórios, além dos resíduos produzidos no ambiente domiciliar, provenientes do cuidado com a saúde, como autoadministração de insulina, cuidados recuperativos e diálises ${ }^{(5)}$. No ambiente hospitalar, a geração de RSS é um resultado inevitável dos cuidados prestados ao paciente, e o aumento do número de instituições para atender à demanda social aumenta a preocupação sobre o destino desses materiais, principalmente pelo crescente uso de materiais descartáveis, sendo primordial a adequada gestão dos RSS para a redução dos riscos existentes ${ }^{(6,7)}$.

A má gestão dos resíduos hospitalares representa um grande risco para a população, pois o manejo inadequado e impróprio pode acarretar sérias consequências para a saúde pública com impacto significativo na degradação do meio ambiente ${ }^{(6,8)}$. Acredita-se que $75 \%$ a $90 \%$ dos resíduos produzidos pelos profissionais de saúde não apresentam risco direto para a população, e que apenas $10 \%$ a $25 \%$ desses resíduos são considerados perigosos e podem ocasionar problemas de saúde ${ }^{(6,8)}$. A falta de conhecimento dos profissionais de saúde e a má gestão hospitalar dos resíduos são os principais fatores que contribuem para a segregação inadequada dos materiais ${ }^{(6,9)}$.

Assim, considerando-se o risco de acidentes de trabalho, doenças ocupacionais e aumento da infecção hospitalar que podem ser gerados pelo incorreto manejo dos RSS, acredita-se que a capacitação dos profissionais de saúde sobre o gerenciamento de resíduos deve acontecer no momento de sua admissão e ser mantido sob supervisão em serviço, objetivando garantir o sucesso do manejo desses resíduos, além de favorecer a redução dos gastos no descarte desses materiais ${ }^{(10)}$.

Tais observações despertaram o interesse de avaliar o conhecimento dos profissionais de saúde que atuam na Unidade de Terapia Intensiva (UTI) em relação à segregação dos resíduos, pois esse setor representa um local onde há demanda elevada de lixo, entretanto, a segregação, muitas vezes, ocorre erroneamente. O conhecimento e a atitude desses profissionais quanto à importância da gestão adequada influencia no modo como o processo é realizado na instituição. Se eles não estão sensibilizados com a gestão correta dos RSS, existe um maior risco iminente para a sociedade relacionado a esses materiais ${ }^{(9)}$.

Diante do exposto, surgiu o seguinte questionamento: a realização de uma ação educativa sobre segregação dos resíduos sólidos com os profissionais de saúde da Unidade de Terapia Intensiva favoreceria a melhora do conhecimento? Acredita-se que este estudo possa subsidiar propostas de intervenção e uma maior conscientização dos profissionais de saúde sobre a temática, pois o desconhecimento e a falta de informação sobre o assunto fazem com que os resíduos sejam ignorados e/ou recebam tratamento inadequado.

Com isso, este estudo teve como objetivo avaliar a ação de estratégia educativa sobre o conhecimento de profissionais que atuam em Unidade de Terapia Intensiva quanto à segregação de resíduos sólidos de saúde.

\section{MÉTODOS}

Estudo experimental, com aplicação de pré e pós-teste com único grupo, desenvolvido no período de fevereiro e 
março de 2015 em uma Unidade de Terapia Intensiva de um hospital público de grande porte localizado na cidade de Fortaleza, Ceará, Brasil.

A amostra compreendeu os profissionais de saúde que estavam na ativa no período da coleta, sendo a seleção por conveniência, conforme os seguintes critérios de inclusão: ser profissional da área da saúde, estar vinculado à instituição e estar de plantão no serviço no momento da coleta de dados e do treinamento proposto. Os critérios de exclusão foram: profissionais que ocupavam exclusivamente cargos de chefia e/ou em serviços administrativos, e profissionais afastados, de férias ou de licença.

A operacionalização da estratégia educativa ocorreu em três momentos: 1) aplicação do pré-teste; 2) estratégia educativa mediada por álbum seriado; 3) aplicação do pósteste após 15 dias da estratégia.

O pré e pós-teste eram semelhantes, representados por um formulário com 33 itens correlacionados com os cinco tipos de lixeiras existentes para descarte (químico, infectante, perfurocortante, comum e reciclável), sendo construído pelos autores com base no referencial teórico da Resolução da Diretoria Colegiada (RDC) 306/2004 e da resolução do Conselho Nacional do Meio Ambiente (CONAMA) $358 / 2005^{(11,12)}$, e com auxílio de três enfermeiras especialistas em UTI.

$\mathrm{O}$ segundo momento do estudo correspondeu à aplicação da estratégia educativa, implementada com o auxílio de álbum seriado sobre segregação de resíduos sólidos em saúde, o qual foi elaborado para este estudo, tendo como referência a experiência profissional dos pesquisadores e a literatura pertinente ao objeto de estudo, sendo realizada uma única vez durante o período de plantão, em uma sala reservada dentro da UTI, e com duração média de 30 minutos. O referido álbum seriado é composto de imagens coloridas e constam definições dos tipos de resíduos, local apropriado para o descarte e as principais resoluções vigentes do país.

Durante a coleta de dados, o pesquisador principal comparecia à UTI nos três períodos do dia (manhã, tarde e noite) e realizava o convite ao profissional de saúde para participar do estudo, sendo solicitada a assinatura do Termo de Consentimento Livre e Esclarecido, garantindo-lhes o anonimato e o sigilo das informações. Após o aceite, era aplicado o pré-teste e, em seguida, realizada a estratégia educativa de forma individual. O pesquisador apresentava o álbum seriado para cada profissional e explicava todos os tipos de resíduos. Ao fim da apresentação, o pesquisador ficava disponível para esclarecimentos de dúvidas e lembrava sobre a aplicação do pós-teste após 15 dias.

Utilizou-se o Excel, versão 8.0, para tabulação dos dados, e o Statistical Package for Social Sciences -
SPSS, versão 17.0, para análise estatística com nível de significância de $p<0,05$. Utilizaram-se medidas de tendência central (média) e dispersão (desvio padrão), além da análise de frequência absoluta (n) e relativa (\%). Na comparação das médias entre o total de acertos no pré e pós-teste, foi usado o teste $t$ independente. Para avaliar se houve uma quantidade de acertos sobre cada tipo de resíduo entre os profissionais, utilizou-se o teste binomial, em que valores de $p$ superiores a 0,05 indicavam porcentagem de acertos não inferior a $70 \%$, sendo estatisticamente significante.

O presente estudo obedeceu aos aspectos éticos segundo as Diretrizes e Normas de Pesquisa em Seres Humanos, dispostas na Resolução n ${ }^{\circ}$ 466/12 do Conselho Nacional de Saúde, sendo aprovado pelo Comitê de Ética em Pesquisa da Instituição em estudo, sob o parecer n ${ }^{\circ} .950795$.

\section{RESULTADOS}

Participaram do pré-teste 41 profissionais de saúde, porém, na aplicação do pós-teste, houve uma perda amostral devido à falta de comparecimento dos profissionais no dia combinado ou recusa em participar devido ao tempo disponível durante o plantão, ficando a amostra final constituída por 28 profissionais.

Tanto no grupo do pré-teste como do pós-teste observou-se um predomínio de mulheres, com frequências de $75,6 \%(\mathrm{n}=31)$ e $89,3 \%(\mathrm{n}=25)$, respectivamente. Os profissionais possuíam os seguintes cargos: fisioterapeuta, enfermeiro, médico, farmacêutico, psicólogo, técnico e auxiliar de enfermagem.

A Tabela I apresenta a pontuação mínima e máxima e as médias obtidas pelos profissionais de saúde no pré e pósteste em relação ao total de acertos sobre os resíduos sólidos em saúde.

Com a realização da estratégia educativa, observou-se uma diferença positiva entre as médias do total de acertos no pré e pós-teste, tanto em valores absolutos $(\mathrm{p}=0,001)$ quanto relativos $(\mathrm{p}=0,001)$.

Nas Tabelas II e III, podem ser observadas as frequências de acertos de cada tipo de resíduo avaliado no pré e pós-teste.

De acordo com a Tabela II, observou-se que, no préteste, os principais erros relacionados à segregação de resíduos infectantes estão associados aos materiais do tipo luvas cirúrgicas estéreis e equipos de BIC, macrogotas ou fotossensíveis. Porém, no pós-teste, os profissionais ainda apresentaram um baixo nível de conhecimento sobre a segregação desses materiais infectantes, principalmente sobre peças anatômicas, bolsa de estomias, drenos, cateteres e sondas, luvas cirúrgicas estéreis e equipos de BIC, macrogotas ou fotossensíveis. 
Tabela I - Comparação do total de acertos antes e após estratégia educativa. Fortaleza, Ceará, 2015.

\begin{tabular}{lccccccc}
\hline Acertos & Grupo & n & Mínimo & Máximo & Média & Desvio Padrão & p-valor \\
\hline \multirow{2}{*}{ Total de acertos do questionário (n) } & Pré-teste & 41 & 13 & 26 & 19,6 & 3,2 & $0,001^{*}$ \\
& Pós-teste & 28 & 18 & 29 & 24,1 & 2,8 & \\
\multirow{2}{*}{ Total de acertos do questionário (\%) } & Pré-teste & 41 & 39,4 & 78,8 & 59,5 & 9,7 & $0,001^{*}$ \\
& Pós-teste & 28 & 54,5 & 87,9 & 73,0 & 8,5 & \\
\hline
\end{tabular}

${ }^{*}$ Teste $t$ para amostras independentes, significativo ao nível de $1 \%$.

Tabela II - Comparação dos acertos no pré e pós-teste sobre resíduo infectante e comum. Fortaleza, Ceará, 2015.

\begin{tabular}{lcccc}
\hline Variáveis & \multicolumn{2}{c}{ Pré-teste } & \multicolumn{2}{c}{ Pós-teste } \\
\cline { 2 - 5 } & $\mathbf{n ~ ( \% )}$ & $\mathbf{p} \dagger$ & $\mathbf{n}(\%)$ & $\mathbf{p}_{\dagger}^{\dagger}$ \\
\hline Resíduo infectante & & & & \\
Equipos de BIC, macrogotas ou fotossensível & $15(36,6)$ & $<0,000$ & $4(14,3)$ & $<0,000$ \\
Bolsa de sangue e derivados & $36(87,8)$ & 0,998 & $24(82,2)$ & 0,984 \\
Fraldas de paciente em isolamento de contato & $39(95,1)$ & 1 & $26(92,9)$ & 0,999 \\
Resíduos produzidos no cuidado com paciente em isolamento & $38(92,7)$ & 0,999 & $28(100)$ & 1 \\
Peças anatômicas & $30(73,2)$ & 0,725 & $18(64,3)$ & 0,317 \\
EPIs utilizados no cuidado com paciente em isolamento & $39(95,1)$ & 1 & $27(96,4)$ & 1 \\
Bolsa de estomias & $15(36,6)$ & 0,998 & $17(60,7)$ & 0,191 \\
Drenos, cateteres e sondas & $34(82,9)$ & 0,980 & $16(57,1)$ & 0,102 \\
Luva cirúrgica estéril & $26(63,4)$ & 0,223 & $12(42,9)$ & 0,002 \\
Resíduo comum & & & & \\
Fralda contendo fezes & $6(14,6)$ & $<0,000$ & $25(89,3)$ & 0,996 \\
Fralda contendo urina & $8(19,5)$ & $<0,000$ & $27(96,4)$ & 1 \\
Gazes utilizadas na antissepsia do paciente & $10(24,4)$ & $<0,000$ & $25(89,3)$ & 0,996 \\
Restos alimentares & $36(87,8)$ & 0,998 & $26(92,9)$ & 0,999 \\
Guardanapos & $34(82,9)$ & 0,980 & $22(78,6)$ & 0,887 \\
Absorventes contendo menstruação & $6(14,6)$ & $<0,000$ & $22(78,6)$ & 0,887 \\
Algodão utilizado para banho no leito & $19(46,3)$ & 0,001 & $27(96,4)$ & 1 \\
Gazes com matéria orgânica contida & - & - & $17(60,7)$ & 0,191 \\
Máscara, luvas de procedimento, propés e gorros & $25(61)$ & 0,138 & $24(82,2)$ & 0,984 \\
Folhas de papel toalha & $32(78)$ & 0,905 & $22(78,6)$ & 0,887 \\
Eletrodos & $26(63,4)$ & 0,223 & $26(92,9)$ & 0,999 \\
\hline
\end{tabular}

* Frequência e porcentagem de acertos do total de profissionais de saúde avaliados antes e depois da intervenção; †Teste binomial. BIC = Bomba de Infusão Contínua; EPI = Equipamento de Proteção Individual.

A categoria sobre os resíduos comuns foi a que teve um maior número de erros entre os profissionais de saúde, os quais foram sobre fralda contendo fezes ou urina; gazes utilizadas na antissepsia do paciente; absorventes contendo menstruação; algodão utilizado para banho no leito; gazes com matéria orgânica contida; máscara, luvas de procedimento, propés e gorro; e eletrodos (Tabela II).

Os profissionais de saúde investigados também apresentaram baixo nível de conhecimento $(<70 \%)$ sobre os resíduos químicos. Quanto aos materiais perfurocortantes, os erros foram sobre a segregação correta do fio-guia e cateteres e do frasco-ampola de vacinas, com 58,5\% e 31,7\% de acertos, respectivamente. Com relação aos resíduos recicláveis, não houve melhora significativa no nível de conhecimento dos profissionais quantos aos materiais, como copo, talheres e tigelas de plástico utilizadas pelo paciente; frascos secos de água destilada e soro fisiológico; invólucro de equipos macrogotas e de BIC; e invólucro de seringas e agulhas (Tabela III). 
Tabela III - Comparação dos acertos no pré e pós-teste sobre resíduo químico, perfurocortante e reciclável. Fortaleza, Ceará, 2015.

\begin{tabular}{lcccc}
\hline \multirow{2}{*}{ Variáveis } & \multicolumn{2}{c}{ Pré-teste } & \multicolumn{2}{c}{ Pós-teste } \\
\cline { 2 - 5 } & $\mathbf{n ~ ( \% )}$ & $\mathbf{p} \dagger$ & $\mathbf{n}(\mathbf{\%})$ & $\mathbf{p} \dagger$ \\
\hline Resíduo químico & & & & \\
$\quad$ Seringa + agulha utilizada no preparo das medicações & - & - & $1(3,6)$ & $<0,000$ \\
$\quad$ Frascos e ampolas de medicamentos & $15(36,6)$ & $<0,000$ & $15(53,6)$ & 0,049 \\
Resíduo perfurocortante & & & & \\
$\quad$ Agulhas para verificar a glicemia & $41(100)$ & 1 & $27(96,4)$ & 1 \\
$\quad$ Seringa + agulha utilizada para injeção intramuscular & $38(92,7)$ & 0,999 & $26(92,9)$ & 0,999 \\
$\quad$ Seringa + agulha utilizada para injeção subcutânea & $39(95,1)$ & 1 & $28(100)$ & 1 \\
$\quad$ Lâminas de bisturi & $40(97,6)$ & 1 & $28(100)$ & 1 \\
$\quad$ Lâminas de barbear & $36(87,8)$ & 0,998 & $26(92,9)$ & 0,999 \\
$\quad$ Fio-guia de cateteres & $24(58,5)$ & 0,078 & $25(89,3)$ & 0,996 \\
$\quad$ Frasco-ampola de vacinas & $13(31,7)$ & $<0,000$ & $7(25)$ & $<0,000$ \\
Resíduo reciclável & & & & \\
$\quad$ Copo, talheres e tigelas de plástico utilizadas pelo paciente & $15(36,6)$ & $<0,000$ & $11(39,3)$ & 0,000 \\
$\quad$ Frascos secos de água destilada e soro fisiológico & $25(61)$ & 0,138 & $20(71,4)$ & 0,635 \\
$\quad$ Invólucro de equipos macrogotas e de BIC & $14(34,1)$ & $<0,000$ & $13(46,4)$ & 0,007 \\
$\quad$ Invólucro de seringas e agulhas & $13(31,7)$ & $<0,000$ & $16(57,1)$ & 0,102 \\
\hline
\end{tabular}

* Frequência e porcentagem de acertos do total de profissionais de saúde avaliados antes e depois da intervenção; †Teste binomial. BIC $=$ Bomba de Infusão Contínua.

\section{DISCUSSÃO}

Saúde pública e promoção da saúde estão interligadas na abordagem e na compreensão dos principais problemas de saúde da população. A saúde pública é vista como a ciência e a arte de prevenir doenças, promover a saúde e prolongar a vida por meio de esforços coletivos organizados entre as sociedades em que se estabelecem condições para que indivíduos e grupos vivenciem uma vida saudável a partir de ações que envolvem educação, habitação, condições de trabalho e políticas públicas, entre outros determinantes ${ }^{(13)}$.

O campo da promoção da saúde é concebido como um novo e promissor paradigma na saúde, dada a amplitude de suas abordagens teórico-metodológicas. Para a Organização Mundial de Saúde (OMS), a promoção da saúde pode ser definida como uma estratégia que tem como objetivo capacitar indivíduos, grupos e comunidades para controlar e melhorar os fatores que interferem na sua saúde, melhorando a qualidade de vida ${ }^{(14)}$.

É irônico pensar que as instituições de saúde, responsáveis pela restauração e manutenção da saúde da comunidade, também ameaçam o bem-estar da população ${ }^{(6,8)}$. Os resíduos ameaçam a sobrevivência dos seres humanos e outros seres vivos, bem como todos os recursos naturais que são necessários para a existência humana ${ }^{(15)}$.

A preocupação com a segregação de RSS é relativamente recente e vem tendo maior ênfase nos serviços de saúde, devido ao surgimento de leis específicas ${ }^{(7)}$. O instrumento utilizado na atual pesquisa para a verificação do nível de conhecimento dos profissionais de saúde foi baseado nas resoluções do Brasil, como a Resolução da Diretoria Colegiada (RDC) 306/2004 e a resolução do Conselho Nacional do Meio Ambiente (CONAMA) 358/2005(11,12). E mesmo sendo baseado em documentos importantes, observou-se no pré-teste um elevado número de erros quanto à classificação dos resíduos.

É importante que todos os profissionais de saúde tenham conhecimento sobre as normas e leis que regem a coleta, a manipulação e a segregação dos $\operatorname{RSS}^{(16)}$. No Brasil, as resoluções $n^{\circ}$ 306/2004 da Agência Nacional de Vigilância Sanitária e a no 358/2005 do CONAMA, bem como a publicação da Lei Federal no 12.305/2010, representam um divisor importante na maneira de conduzir o gerenciamento desse tipo de resíduo ${ }^{(11,12)}$.

Assim, a gestão de RSS é um conjunto de procedimentos planejados e implementados, baseados em evidências científicas e técnicas que visam minimizar a produção de lixo, bem como proporcionar um método de eliminação segura e eficiente para os resíduos gerados. Ou seja, não visa apenas a proteção dos pacientes e profissionais de saúde, mas também a preservação da saúde pública, dos recursos naturais e do meio ambiente ${ }^{(7)}$.

Tal gestão varia em cada país, de acordo com vários fatores, como condições socioeconômicas, nível de educação, recursos disponíveis, tecnologias de tratamento e sistemas de monitoração de práticas inadequadas ${ }^{(17)}$. $\mathrm{O}$ objetivo da 
gestão de RSS é a adequada segregação, coleta, transporte, manuseio e descarte, de modo a garantir a segurança dos pacientes, dos profissionais e do meio ambiente ${ }^{(9)}$, visando reduzir o impacto sobre a saúde pública, com melhoria da sustentabilidade ambiental e econômica do sistema de cuidados de saúde ${ }^{(17)}$

A segregação de resíduos é o passo mais crucial para a gestão adequada dos RSS, e a segregação correta em locais apropriados deve ser uma preocupação constante dos profissionais de saúde ${ }^{(16,18)}$. A falta de conhecimento sobre o gerenciamento dos resíduos sólidos encontrado no presente estudo pode ser umas das causas para a ocorrência do descarte inadequado dos materiais hospitalares. Seria necessário que os profissionais de saúde tenham acesso às sessões de educação continuada sobre os diversos problemas e perigos encontrados em sua área de trabalho, inclusive o manuseio adequado dos RSS.

O descarte inadequado dos RSS está relacionado a dois grandes problemas: o impacto ambiental devido a uma grande variedade de produtos perigosos e o potencial risco infeccioso desses materiais durante a sua manipulação(16). Assim, os materiais devem ser alocados em vários recipientes com cores diferentes, devendo haver caixotes de lixo em número suficiente, em locais e cores adequados, para que os profissionais de saúde desprezem corretamente os materiais ${ }^{(18)}$. Com isso, os serviços de saúde devem fornecer recipientes apropriados para acondicionar cada tipo de resíduo, garantir identificação satisfatória do material, facilitar as atividades de limpeza e transporte, além de fornecer sacos plásticos específicos para cada grupo de resíduos $^{(19)}$. No presente estudo, observou-se que, mesmo após a estratégia educativa, ainda houve erros no descarte de materiais perfurocortantes e infectantes, embora tenham sido pequenos.

Os resíduos hospitalares são divididos em cinco categorias, e o método mais comum de segregação é a utilização de sacos com códigos de cores. Assim, os profissionais de saúde devem garantir que recipientes com sacos adequados sejam usados para o recolhimento do lixo hospitalar, tendo em vista que a má gestão dos resíduos pode gerar riscos ambientais, biológicos e radiológicos para a população ${ }^{(6,8,20)}$

Estudo de intervenção realizado no Paquistão, com o objetivo de avaliar o gerenciamento dos resíduos em dois hospitais terciários, com 275 profissionais de saúde, demonstrou que o treinamento é uma intervenção eficaz, sendo comprovada pela diferença estatisticamente significante entre o pré e pós-teste sobre conhecimento, atitude e prática ${ }^{(21)}$. Isso também foi observado no presente estudo, pois houve uma melhora do conhecimento dos profissionais de saúde após a intervenção educativa.
Outro estudo utilizou um questionário autoaplicável para identificar conhecimento, atitude e prática entre os profissionais de saúde sobre RSS, o qual evidenciou a importância de incluir essa temática nos currículos de graduação. $\mathrm{O}$ desenvolvimento de cursos de curta duração e/ou seminários melhora a adesão à vacinação, ao uso de Equipamentos de Proteção Individual (EPI) e ao manuseio do resíduo hospitalar ${ }^{(22)}$.

O presente estudo também demonstrou uma mudança significativa no nível de conhecimento dos participantes envolvidos. A melhora do conhecimento desses profissionais após a capacitação evidencia que a implementação da educação continuada representa a oportunidade de os profissionais atualizarem seus conhecimentos e melhorarem a prática assistencial do serviço de saúde.

Sabe-se que a gestão eficaz dos resíduos não é apenas uma necessidade legal do ambiente hospitalar, mas também uma responsabilidade social. Assim, há uma necessidade de recursos materiais e educação continuada para auxiliar os administradores e profissionais de saúde na realização dessa tarefa. Com isso, é essencial que a instituição implemente um programa de educação continuada para todos os funcionários, a fim de clarificar as definições e segregação de resíduos, além de um sistema de monitoramento e vigilância da gestão dos $\operatorname{RSS}^{(18)}$. Isso possibilita tornar a equipe capacitada e melhorar a eficácia e eficiência da atenção prestada aos pacientes e comunidade ${ }^{(23)}$.

Os RSS são todos aqueles materiais resultantes de atividades exercidas nos serviços de atenção à saúde e que, por suas características, necessitam de processos diferenciados em seu manejo, exigindo ou não tratamento prévio até sua disposição final. Os RSS são subdivididos em cinco grupos: A- infectantes; B- químicos; C- radioativos; D- comuns; e E- perfurocortantes ${ }^{(12)}$.

A segregação incorreta pode ser a principal responsável pelos elevados custos com transporte e destinação final do lixo. Essa prática de segregação incorreta dos resíduos, principalmente do acondicionamento em conjunto dos Grupos A e D, evidencia a escassez de conhecimento dos profissionais de saúde sobre o manejo dos RSS, gerando um aumento substancial no custo para o tratamento dos resíduos $^{(24,25)}$.

A falta de recursos financeiros e humanos e a falta de controle na segregação dos resíduos são os principais problemas encontrados no gerenciamento desses materiais ${ }^{(16)}$. Um estudo realizado recentemente em um município brasileiro ${ }^{(25)}$ mostrou que a segregação correta dos RSS do Grupo D e A reduziria a quantidade de resíduos do Grupo A, gerando redução de custos, com incineração em até $57 \%$. Além de sugerir que, para melhorar o Plano de Gerenciamento de Resíduos de Serviços de Saúde (PGRSS), são necessários capacitação profissional, estrutura física e 
material para um acondicionamento apropriado dos RSS, a fim de que haja o cumprimento da RDC n ${ }^{\circ} 306 / 2004$ e Resolução nº 358/2005 do CONAMA.

A gestão de resíduos bem-sucedida representa um desafio para os países, devido ao investimento financeiro insuficiente, baixo conhecimento e falta de consciência dos profissionais e ausência de controle eficaz dos resíduos ${ }^{(15)}$. A educação permanente é um instrumento fundamental na mudança de paradigma dos profissionais de saúde e corrobora para uma excelência no atendimento, redução de custos e diminuição do impacto socioambiental. É de responsabilidade dos estabelecimentos de saúde promover aulas e práticas de conscientização sobre resíduos de saúde que resultem na minimização de gastos desnecessários com incineração e possibilitem a redução de custos com disposição final, como preconiza a legislação vigente no país.

Verificou-se no presente estudo que a maior porcentagem de erros esteve relacionada com os resíduos infectantes, químicos, comuns e recicláveis. Sabe-se que, na fase de segregação dos RSS, pode haver confusão e conceitos errôneos sobre a classificação de cada material, o que pode ser solucionado com ações educativas e treinamentos sobre a temática ${ }^{(26)}$. Resíduos comuns contendo secreções como fralda contendo fezes, fralda contendo urina, gazes utilizadas na antissepsia do paciente, absorventes contendo menstruação, algodão utilizado para banho no leito e gazes com matéria orgânica contida apresentaram os maiores índices de erros no pré-teste. Isso pode estar relacionado com a falta de conhecimento dos profissionais para classificar esses materiais que apresentam secreções, causando confusão com resíduos infectantes.

A principal preocupação com os RSS é devido à presença de organismos patogênicos e substâncias orgânicas de efeito adverso para a saúde da população. Alguns resíduos podem conter um número significativo de organismos, incluindo estirpes virulentas de vírus e bactérias patogênicas ${ }^{(8)}$. Assim, os resíduos infectantes são considerados o segundo grupo de maior risco em todo o mundo, os quais merecem atenção da equipe de saúde durante a sua segregação, pois o manejo inadequado deles pode ocasionar infecções aos trabalhadores de saúde, como hepatites B e C, febre tifoide, cólera, tuberculose, infecções de pele, infecções respiratórias e HIV/AIDS ${ }^{(21)}$. No atual estudo, os resíduos infectantes que apresentaram maior número de erros na sua classificação pelos profissionais de saúde foram: equipos, bolsa de estomias e luva cirúrgica estéril.

Os resíduos químicos são representados por materiais contendo substâncias químicas que podem apresentar risco à saúde da população ou ao meio ambiente, dependendo de suas características de periculosidade ${ }^{(27)}$. A regulamentação brasileira sobre RSS descrimina que os resíduos do Grupo B (químicos), quando segregados e acondicionados corretamente, podem ser devolvidos ao fabricante/ fornecedor do produto químico ${ }^{(11,12)}$.

Verificou-se que a maior parte dos profissionais investigados no presente estudo apresentou baixo conhecimento sobre os resíduos químicos, pois poucos classificaram os materiais "seringa e agulha utilizada no preparo das medicações" e "frascos e ampolas de medicamentos" como resíduos químicos. Infere-se também que, mesmo após a intervenção educativa, não houve uma melhora significativa do conhecimento dos profissionais sobre tais materiais.

Dentre todos os riscos ocupacionais relacionados à má gestão dos RSS, há uma preocupação especial com a ocorrência de acidentes com materiais biológicos contendo os vírus HIV, hepatites $\mathrm{B}$ e C, para os quais existe uma forte evidência de transmissão através de resíduos de saúde ${ }^{(8)}$. A segregação segura de materiais perfurocortantes é uma prioridade fundamental, devido à sua classificação como resíduos altamente perigosos, e a gestão correta desses materiais tem impacto direto na redução de acidentes ocupacionais ${ }^{(17)}$.

A manipulação de materiais perfurocortantes, como agulhas, cateter venoso e lâminas de bisturi, utilizados durante a assistência ao paciente, é vista como a principal causa de acidentes ocupacionais entre os profissionais de saúde ${ }^{(28)}$. Para os resíduos perfurocortantes, o descarte correto é efetivado quando o objeto é desprezado em um recipiente com proteção e paredes rígidas ${ }^{(26)}$. Dos materiais dispostos no teste de conhecimento, apenas resíduos como fio-guia de cateteres e frasco-ampola de vacinas apresentaram baixo índice de acertos pelos profissionais investigados no presente estudo.

Em qualquer serviço ou nível de atenção à saúde, a qualidade da assistência prestada à comunidade depende do gerenciamento adequado dos resíduos gerados, o que exige a presença de profissionais capacitados e comprometidos com essa atividade ${ }^{(29)}$. Destaca-se que os resíduos advindos dos serviços de saúde podem se tornar graves problemas para a sociedade quando manejados de forma inadequada, contribuindo para a degradação ambiental e riscos à saúde da comunidade. É primordial que profissionais de saúde, gestores e instituições sejam sensibilizados quanto à responsabilidade individual e coletiva com a gestão adequada dos $\operatorname{RSS}^{(29)}$.

A capacitação realizada durante o período de trabalho com os profissionais que atuam na UTI favoreceu o aumento do conhecimento dos profissionais sobre a segregação dos RSS, o que poderá favorecer a redução de acidentes ocupacionais de saúde e a redução dos gastos com materiais.

Como limitação deste estudo, salienta-se a impossibilidade de intervenção no PGRSS do Hospital Universitário para adequação das lixeiras conforme 
normatizações brasileiras sobre RSS, devido à escassez de tempo hábil, tendo em vista que, neste estudo, foi possível realizar apenas a avaliação do conhecimento sobre RSS, com o intuito de sensibilizar a equipe de saúde para a atenção na gestão eficaz dos materiais, principalmente no momento da segregação.

Sabe-se que gerenciamento dos resíduos engloba um conjunto de procedimentos de gestão, planejamento e implementação a partir de bases científicas e técnicas, normativas e legais, com o objetivo de minimizar a produção de resíduos e proporcionar um encaminhamento seguro aos mesmos. Com isso, os profissionais de UTI saúde são responsáveis por uma das etapas mais importantes do gerenciamento dos resíduos, que é a segregação, sendo essencial que o serviço possibilite periodicamente cursos de capacitação para que esses profissionais tenham conhecimento sobre a classificação correta de cada material.

Sugere-se a realização de outros estudos que visem acompanhar por um maior período os efeitos obtidos com a intervenção educativa e associar o aumento do conhecimento dos profissionais com a redução do peso do lixo hospitalar.

\section{CONCLUSÃO}

Observou-se que a estratégia educativa proporcionou o aumento do conhecimento dos profissionais sobre a segregação dos resíduos, principalmente com relação aos materiais comuns, recicláveis e infectantes. Tal resultado poderá favorecer a redução de acidentes ocupacionais de saúde e a redução dos gastos com materiais.

\section{REFERÊNCIAS}

1. Whitehead D. Health promotion in the practice setting: findings from a review of clinical issues. Worldviews Evid Based Nurs. 2006;3(4):165-84.

2. Richard L, Gendron S, Beaudet N, Boisvert N, Soleil MS, Brodeur MHG. Health Promotion and Disease Prevention Among Nurses Working in Local Public Health Organizations in Montréal, Québec. Public Health Nurs. 2010;27(5):450-8.

3. Makajic-Nikolica D, Petrovica N, Belica A, Rokvica M, Radakovica JA, Tubicb V. The fault tree analysis of infectious medical waste management. J Clean Prod. 2016;113:365-73.

4. Windfeld ES, Brooks MS. Medical waste management: a review. J Environ Manage. 2015;163:98-108.

5. Prüss A, Giroult E, Rushbrook P. Safe management of wastes from health-care activities [Internet]. Geneva:
World Health Organization; 2014 [acesso em 2015 Mar 3]. Disponível em: http://www.searo.who.int/srilanka/ documents/safe_management_of_wastes_from healthcare_activities.pdf

6. Sengodan VC. Segregation of biomedical waste in an South Indian tertiary care hospital. J Nat Sci Biol Med. 2014;5(2):378-82.

7. Diaz PS, Soares SGA, Camponogara S, Saldanha VS, Menegat RP, Rossato GC. Waste Management: A descriptive-exploratory study in the emergency room of a teaching hospital. Online Braz J Nurs (Online) [Internet]. 2013 [acesso em 2015 Mar 3];12(4):964-74. Disponível em: http://www.objnursing.uff.br/index. $\mathrm{php} /$ nursing/article/view/4090

8. Chudasama RK, Sheth A, Rangoonwala M, Joshi N, Zalavadiya D, Bhola C. Awareness and practice of biomedical waste management among different health care personnel at Tertiary Care Centre, Rajkot, India. Online J Health Allied Scs. 2014;13(1):1-6.

9. Kishore J, Agarwal R, Kohli C, Sharma PK, Kamat NV, Tyagi SC. Status of Biomedical Waste Management in Nursing Homes of Delhi, India. J Clin Diagn Res. 2014;8(3):56-8.

10. Nunes TSP, Gutemberg ACB, Armando CB, Pinto FF, Lemos MC, Passos JP. Geren- ciamento de resíduos de serviços de saúde: uma revisão de literatura. Rev Pesqui Cuid Fundam (Online) [acesso em 2015 Mar 12]. 2012;4(Supl 1):57-60. Disponível em: http://www. seer.unirio.br/index.php/cuidadofundamental/article/ view/1697/pdf_555

11. Ministério da Saúde (BR), Agência Nacional de Vigilância Sanitária. Resolução $\mathrm{n}^{\circ} .306$ de 7 de dezembro de 2004. Regulamento técnico para o gerenciamento de resíduos dos serviços de saúde [Internet]. Brasília: Ministério da Saúde; 2004 [acesso em 2015 Mar 3]. Disponível em: http://portal.anvisa. gov.br/wps/wcm/connect/10d6dd00474597439fb6df3 $\mathrm{fbc} 4 \mathrm{c} 6735 / \mathrm{RDC}+\mathrm{N} \% \mathrm{C} 2 \% \mathrm{BA}+306,+\mathrm{DE}+7+\mathrm{DE}+\mathrm{DEZ}$ EMBRO+DE+2004.pdf

12. Ministério da Saúde (BR), Conselho Nacional do Meio Ambiente. Resolução n 358/05. Dispõe sobre o tratamento e a disposição final dos resíduos dos serviços de saúde e dá outras providências [Internet]. Brasília: Ministério da Saúde; 2005 [acesso em 2015 Mar 3]. Disponível em: http://www.mma.gov.br/port/ conama/res/res05/res35805.pdf

13. Povlsen L, Borup I. Health Promotion: A developing focus area over the years. Scand J Public Health. 2015;43(16 Suppl):46-50. 
14. World Health Organization. What is health promotion? [acesso em 2016 Maio 5]. Disponível em: www.who. int/healthpromotion/en/

15. Hossain MS, Santhanam A, Nik Norulaini NA, Omar AK. Clinical solid waste management practices and its impact on human health and environment: a review. Waste Manag. 2011;31(4):754-66.

16. Oliveira LL, Souza PM, Clementino FS, Paiva SC, Rocha FDLJ. Resíduos dos serviços de saúde: desafios e perspectivas na atenção primária. Rev Enferm UERJ. 2014;22(1):29-34.

17. Caniato $M$, Tudor $T$, Vaccari $M$. International governance structures for health-care waste management: a systematic review of scientific literature. J Environ Manage. 2015;153:93-107.

18. Ministério da Saúde (BR), Agência Nacional de Vigilância Sanitária. Manual de gerenciamento de resíduos de serviços de saúde [Internet]. Brasília: Ministério da Saúde; 2006 [acesso em 2015 Mar 3]. Disponível em: http://www.anvisa.gov.br/servicosaude/ manuais/manual_gerenciamento_residuos.pdf

19. Ministério da Saúde (BR), Secretaria de Gestão do Trabalho e da Educação na Saúde, Departamento de Gestão da Educação em Saúde. Política Nacional de Educação Permanente em Saúde [Internet]. Brasília: Ministério da Saúde; 2009 [acesso em 2015 Mar 3]. Disponível em: http://bvsms.saude.gov.br/bvs/ publicacoes/politica_nacional_educacao_permanente saude.pdf

20. Chethana T, Thapsey H, Gautham MS, Sreekantaiah P, Suryanarayana SP. Situation analysis and issues in management of biomedical waste in select small health care facilities in a ward under Bruhat Bengaluru Mahanagara Palike, Bangalore, India. J Community Health. 2014;39(2):310-5.

21. Ministério da Saúde (BR), Conselho Nacional do Meio Ambiente. Resolução nº. 358, de 29 de abril de 2005. Sobre o tratamento e a disposição final dos resíduos dos serviços de saúde e outras providências [Internet]. DOU, no 84, 2005 Maio 4, Brasília; 2005. Seção 1:63-65358 [acesso em 2015 Mar 3]. Disponível em: http://www.feam.br/images/stories/2015/RSS/res $\% 20$ conama\%20358\%202005.pdf

22. Kumar R, Somrongthong R, Shaikh RT. Effectiveness of intensive healthcare waste management training model among health professionals at teaching hospitals of Pakistan: a quasi-experimental study. BMC Health Serv Res. 2015; 15:81.
23. Nkonge Njagi A, Mayabi Oloo A, Kithinji J, Magambo Kithinji J. Knowledge, attitude and practice of healthcare waste management and associated health risks in the two teaching and referral hospitals in Kenya. J Community Health. 2012;37(6):1172-7.

24. Basu M, Das P, Pal R. Assessment of future physicians on biomedical waste management in a tertiary care hospital of West Bengal. J Nat Sci Biol Med. 2012;3(1):38-42.

25. Aduan SA, Braga FS, Zandonade E, Salles D, Cussiol NAM, Lange LC. Avaliação dos resíduos de serviços de saúde do Grupo A em hospitais de Vitória (ES), Brasil. Eng Sanit Ambient. 2014;19(2):133-41.

26. Santos MA, Souza AO. Conhecimento de enfermeiros da estratégia Saúde da família sobre resíduos dos serviços de saúde. Rev Bras Enferm. 2012; 65(4):64552.

27. Stehling MC, Cunha LM, Louredo LM, Camargo CG, Haddad JPA, Silva IJ, et al. Gestão de resíduos com risco biológico e perfurocortantes: conhecimento de estudantes de graduação das áreas biológicas e da saúde da Universidade Federal de Minas Gerais. REME Rev Min Enferm. 2013;17(3):601-7.

28. Kumar R, Gupta AK, Aggarwal AK, Kumar A. A descriptive study on evaluation of bio-medical waste management in a tertiary care public hospital of North India. J Environ Health Sci Eng. 2014;12:69.

29. Mendes AA, Veiga TB, Ribeiro TML, André SCS, Macedo JI, Penatti JT, et al . Resíduos de serviços de saúde em serviço de atendimento pré-hospitalar móvel. Rev Bras Enferm. 2015;68(6):1122-9.

\section{Endereço do primeiro autor:}

Bruna Bianchi Bilo

Universidade Federal do Ceará - UFC

Departamento de Enfermagem

Rua Alexandre Baraúna, 1115

Bairro: Rodolfo Teófilo

CEP 60.430-160 - Fortaleza - CE - Brasil

E-mail: brunabbianchi@hotmail.com

\section{Endereço para correspondência:}

Joselany Áfio Caetano

Universidade Federal do Ceará - UFC

Departamento de Enfermagem

Rua Alexandre Baraúna, 1115

Bairro: Rodolfo Teófilo

CEP 60.430-160 - Fortaleza - CE - Brasil

E-mail: joselany@ufc.br 